\title{
Re-explorations in living donor liver transplantation (LDLT): An Egyptian experience in 100 cases. Lessons learned.
}

\author{
Refaat Refaat Kamel, ${ }^{a} M D$ FRCS(Eng); Adel Hosny, ${ }^{b} M D$; Hassan Sallam, ${ }^{c} M D$; \\ Wahid Doss, ${ }^{d}$ MD; Hatem El-Gabaly, ${ }^{c} M D$ \\ a) Unit of GIT and Liver Transplantation, Ain-Shams University, Cairo, Egypt. \\ b) Unit of GIT and Liver Transplantation, Cairo University, Cairo, Egypt. \\ c) Department of Radiology, Cairo University, Cairo, Egypt. \\ d) Department of Tropical Medicine, Cairo University, Cairo, Egypt.
}

\section{Introduction:}

Liver transplantation is the only chance of cure for those with end-stage liver disease.The improvements in surgical technique and immunosuppressive regimens have made liver transplantation a standard treatment for advanced cirrhosis. Organ shortage, law restrictions and sometimes cultural backgrounds have made LDLT the only viable option and a much needed technique in Egypt. The concept of donating a piece of one's liver to save a family member has considerable appeal but reliable information about risks must be provided to potential donors and their family members.

Live donor liver transplantation should offer an overall advantage to the recipient when compared to waiting for an acceptable deceased donor organ to become available for transplantation. The decision to proceed with a live donor liver transplant should be made after a careful analysis of the recipient risk to benefit ratio as it relates to severity of liver failure and quality of life.

The estimated risk of mortality and morbidity currently associated with live donor right hepatectomy is $0.4 \%$ and $35 \%$ respectively. Since the risk to the donor is considerable, programs performing live donor liver transplantation should institute procedures and protocols

that insure that donor mortality and morbidity is minimized.

Over 4years, we have performed 100 cases of adult LDLT in one centre in Egypt (Dar El Fouad Hospital).

The incidence of re-explorations in patients undergoing LDLT is $25-30 \%$. This incidence of re-explorations is extremely high compared with the incidence in other surgical fields. Such re-explorations require familiarity with the operative field of this procedure as well as with the pre- and postoperative management of these patients.

It is worth mentioning that none of our donors required re-exploration.

\section{Lessons:}

- The complication rate for elective surgery in patients who have undergone a previous LDLT is reasonably small, and any procedure can be carried out safely without compromising the graft function.

- Extreme care must be accorded to patients undergoing emergency surgery. As well as the complications of abdominal surgery, these patients have the extra burden of steroid and immunosuppressive therapy and, in many cases, compromised graft function, either due to small graft weight or a not fully regained function.

- Postoperative bleeding following liver transplantation is $10-15 \%$. One-third of cases do not have a point source of bleeding. Bleeding related to coagulopathy is typically slower with the patient more or less stable and with less requirements for volume replacement. Re-operation in these cases is needed to check for major vessel bleeding and also to evacuate haematomas. These can compress hilar structures and compromise the graft blood supply, and also be a potential source of infection.

- Bleeding from the hilum should be approached in a systematic manner; various structures in the hilum are individually clamped to identify the bleeding source. This is the only way to identify the bleeding source in cases of major profuse bleeding. Temporary clamping of the various hilar 
Cases necessitating immediate surgical re-explorations (22\%):

\begin{tabular}{|l|l|}
\hline Cause & Management \\
\hline Burst 3 cases & Repair \\
\hline Thrombosed a. (2 cases) & $\begin{array}{l}\text { Operative Thrombectomy + Urokinase flush via neonatal catheter } \\
\text { (back-bleeding) }\end{array}$ \\
\hline Bleeding cases (9 Cases) & 1- Bile duct bleeding \\
Learning curve & 2- Bleeding omentum \\
& 3- Source had stopped \\
& 4- Liver tear (2) staged approach \\
& 5- Slipped ligature on hepatic artery (selective clamping) \\
& 6- Diaphragm \\
\hline Unseen a. & Positional (doppler) + low threshold for exploration \\
\hline Reduced arterial signal & Spasm, papaverine, return of signal \\
\hline Biliary peritonitis & Drainage (no primary repair) \\
4 cases & Later 2nd exploration for reconstruction \\
& with isolated loop (1 case) \\
\hline Biliary Collection (1 case) & External Surgical Drainage for 2nd stage reconstruction \\
\hline Portal steal phenomenon & Ligation of collateral \\
\hline
\end{tabular}

Delayed surgical re-explorations $(12 \%)$ :

\begin{tabular}{|l|l|l|}
\hline N o & Cause & Management \\
\hline 5 & Incisional hernias & Repair \\
\hline 4 & Biliary strictures & reconstruction \\
\hline 1 & Biliary leak & Reconstruction with isolated loop \\
\hline 1 & Oblique Hernia & Repair \\
\hline 1 & Ileostomy for isolated loop & Closure of ileostomy \\
\hline
\end{tabular}

structures does not have a deleterious effect on the grafted liver.

- Bleeding from liver tears in a severely congested graft should be approached according to the damage control principles, with a staged procedure involving initial packing followed by a second exploration for removal of packs and peritoneal toilet. One should resist any attempt at mobilization of the congested graft as it fractures easily with severe hemorrhage. Minor biliary leaks from the cut surface are expected and these stop spontaneously after few days. If outflow obstruction is ruled out by Doppler study, severe rejection may be the reason for the tense liver. Liver biopsy is thus necessary.

- Cases of biliary peritonitis are treated by peritoneal drainage and toilet with no attempts at repair initially. Localized biliary leaks can be managed by repeated aspirations, pigtail drainage and ERCP stenting.

- Intra-operative cholangiography is an integral part of patient assessment during redo for biliary strictures or for leaked anastomoses. In the latter case, the leakage(s) might not be from the anastomostic site. The donor operative cholangiogram provides vital data for comparison with the intra-operative cholangiogram performed during reexploration.

- A burst abdomen is a purely technical problem. Cases with massive postoperative ascites are more liable to this complication.

- Completion Doppler study of the liver vasculature is mandatory at the end of the procedure, as operative manipulation during 


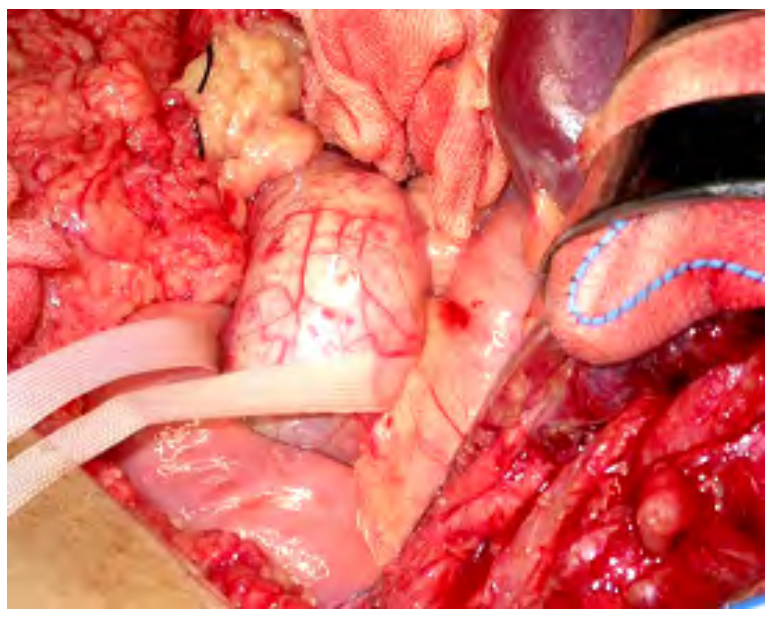

Huge lieno-renal collateral

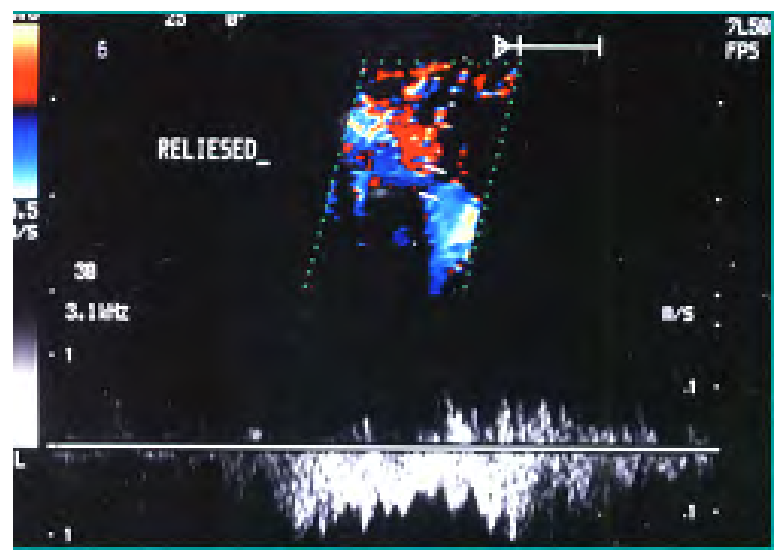

Doppler: Reversed portal flow in the recipient due to steal phenomenon from the huge collateral

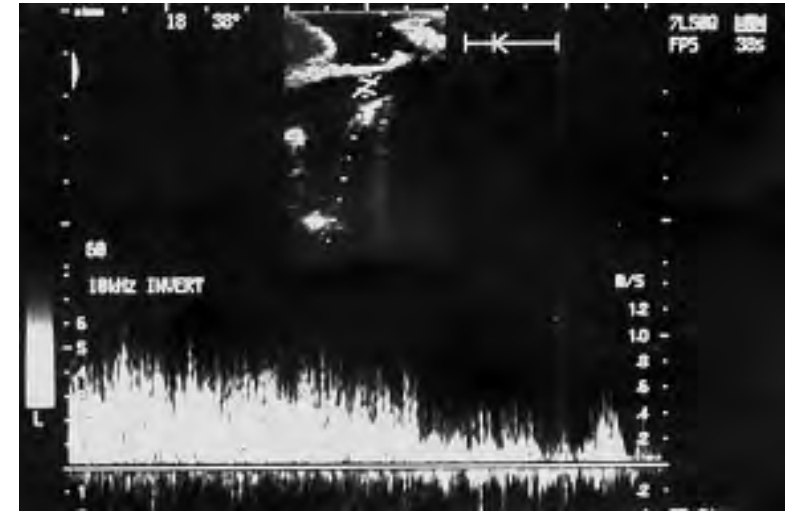

Doppler: Corrected hepatopedal portal flow following ligation of the collateral

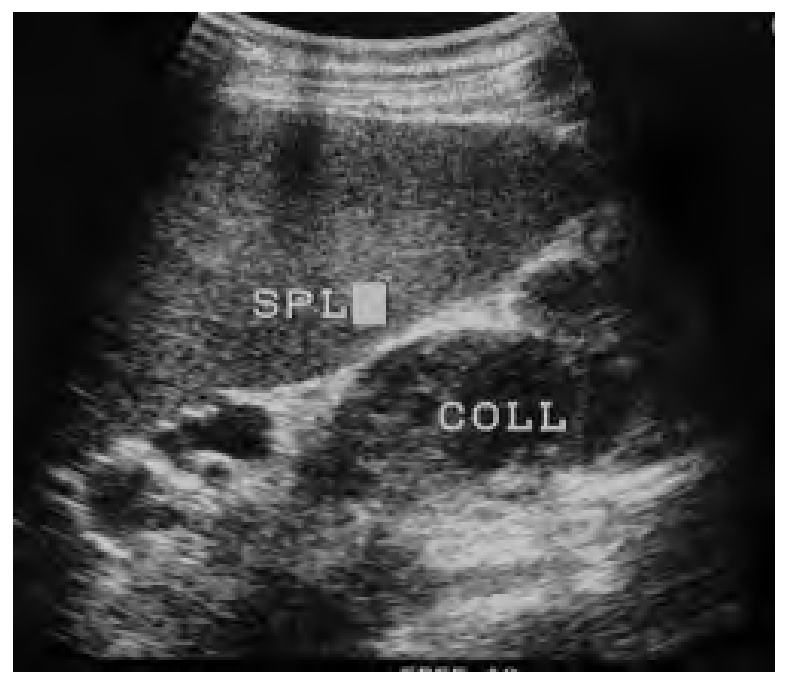

Thrombosed collateral on follow up doppler 

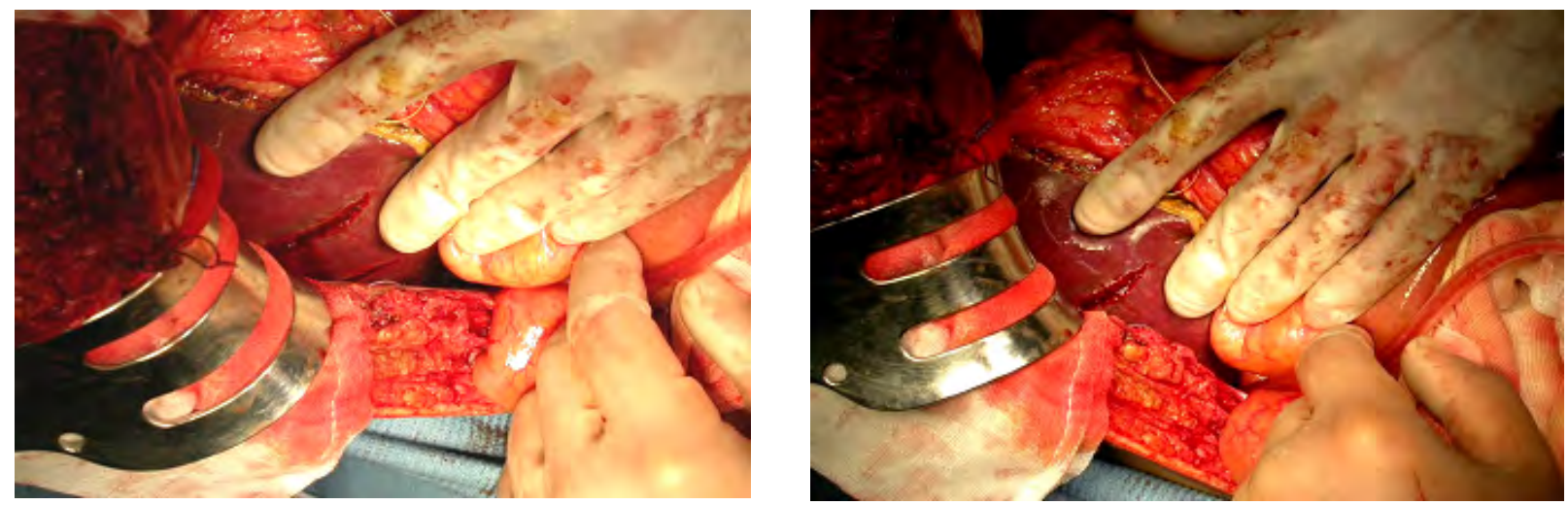

Liver tears in a newly transplanted liver resulting in bleeding and re-exploration and implementation of damage control techniques.

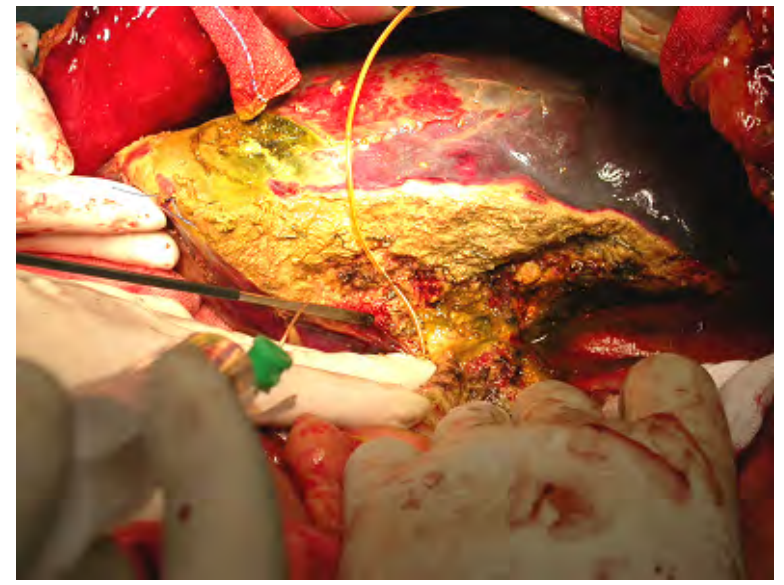

Biliary peritonitis with extensive infection necessitating drainage followed by 2 ry reexploration and staged reconstruction

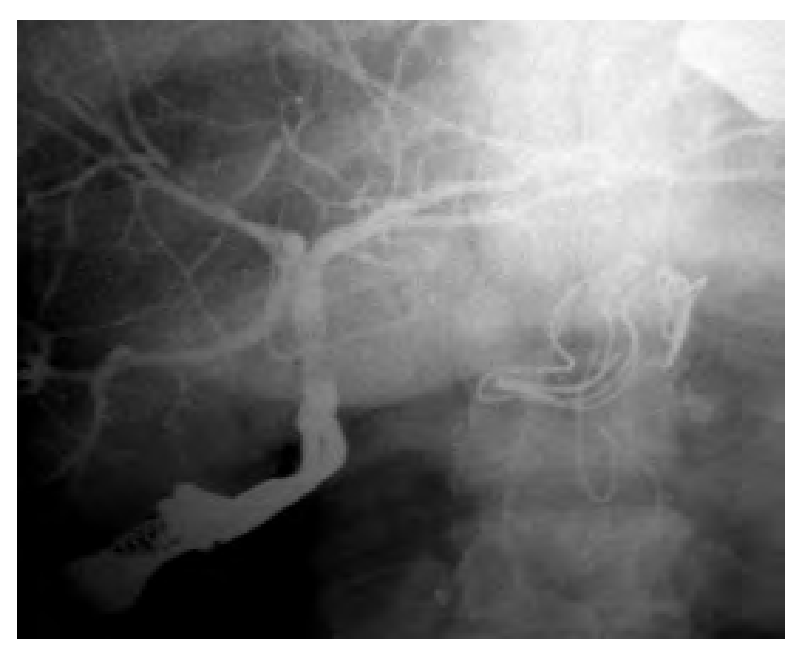

Donor Cholangiogram

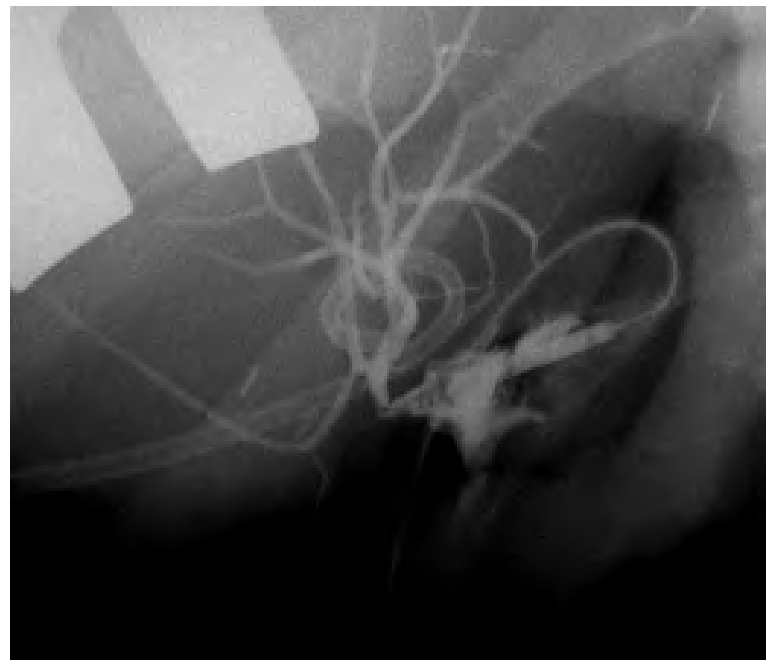

Recipient cholangiogram from same donor in the leaking recipient, making sure there are no missed ducts before final repair is being done. Comparison of the two cholangiograms is extremely important. 
re-exploration or the original pathology that has necessitated the procedure can compromise any of the anastomoses.

- Liver biopsy is an essential step once the abdomen has been entered.

- Large portosystemic collaterals can result in a portal steal phenomenon with a reversal of portal flow into a hepatofugal direction soon after reperfusion of the liver or after few days. In the described case, the completion Doppler at the initial operation did not show reversal of the flow. However, on the next day there was evident hepatofugal flow that was only corrected after exploration of the patient and ligation of the huge lienorenal collateral. This resulted in immediate reversal of the flow with a hepatopedal flow.

- We adopted a policy of early exploration of nonvisualized hepatic arterial signal or a persistently weak signal with eminent occlusion for early salvage of the artery. This policy has proved of great

- Thrombosis of the hepatic artery detected in the first 10 days neces-sitates immediate exploration with thrombectomy and irrigation with thrombolytic agents. Back bleeding from the liver side is a crucial sign after liver irrigation with thrombolytic agents and ensures washing out intrahepatic thrombi.

- Adhesive intestinal obstruction developed few days after LDLT in a patient who had undergone hysterectomy a few years before necessitated re-exploration with the added risk of morbidity. Adhesions due to previous surgery in LDLT patients should be completely dissected even if away from the operative field.

\section{Conclusion:}

The introduction of Living Donor Liver Transplantation (LDLT) in Egypt in 2001 has revolutionized the management of advanced liver disease. It has allowed us to serve many patients but the real scale of transplantation will not be realized unless the law allowing brain death and cadaveric donation is passed.

\section{References:}

1- American Society of Transplant Surgeons, Ethics Committee. Position paper on adultto-adult living donor liver transplantation. Liver Transpl 2000; 6:815-817.

2- Broering DC, Sterneck M, Rogiers X: Living donor liver transplantation. $J$ Hepatol 2003; 38:S119-S135.

3- Brown RS, Russo MW, Lai M, et al: A survey of liver transplantation from living adult donors in the United States. $N$ Engl J Med 2003; 348:818-825.

4- Consensus statement on the live organ donor: report of a national consensus conference on living donor organ transplantation. JAMA 2000; 284:29192926.

5- Schiano TD, Kim-Schluger L, Gondolesi G, Miller CM: Adult living donor liver transplantation: the hepatologist's perspective. Hepatology 2001; 33:3-9.

6- Surman OS: The ethics of partial-liver donation. N Engl J Med 2002; 346:1038.

7- Trotter JF, Wachs M, Everson GT, Kam I: Adult-to-adult transplantation of the right hepatic lobe from a living donor. $N$ Engl J Med 2002; 346:1074-1082. 